\title{
Transient Absorption Spectroscopy of TlBr Crystals Using Pulsed Electron Beams
}

\author{
Masanori Koshimizu, ${ }^{1 *}$ Yusa Muroya, ${ }^{2}$ Shinichi Yamashita, ${ }^{3}$ Mitsuhiro Nogami, ${ }^{4}$ \\ Keitaro Hitomi, ${ }^{4}$ Yutaka Fujimoto, ${ }^{1}$ and Keisuke Asai ${ }^{1}$ \\ ${ }^{1}$ Department of Applied Chemistry, Graduate School of Engineering, Tohoku University, \\ 6-6-07 Aoba, Aramaki, Aoba-ku, Sendai 980-8579, Japan \\ ${ }^{2}$ Institute of Scientific and Industrial Research, Osaka University, 8-1 Mihogaoka, Ibaraki, Osaka 567-0047, Japan \\ ${ }^{3}$ Nuclear Professional School, School of Engineering, The University of Tokyo, \\ 2-22 Shirakata-shirane, Tokai-mura, Naka-gun, Ibaraki 319-1188, Japan \\ ${ }^{4}$ Department of Quantum Science and Energy Engineering, Graduate School of Engineering, Tohoku University, \\ 6-6-01-2 Aoba, Aramaki, Aoba-ku, Sendai 980-8579, Japan
}

(Received December 20, 2019; accepted February 27, 2020)

Keywords: transient absorption, pulse radiolysis, $\mathrm{TlBr}$, carrier transport, semiconductor detector

We analyzed the transient absorption properties of $\mathrm{TlBr}$ crystals using pulsed electron beams as excitation sources. We observed transient absorption spectra and temporal profiles on the pico- and nanosecond scales and compared the results obtained for TIBr crystals that are empirically appropriate with those that are inappropriate for semiconductor detectors. The results showed negligible differences in properties between the two types of crystal, which indicates that their trap center concentrations were similar. A transient absorption band was observed at approximately $1160 \mathrm{~nm}$ on the nanosecond scale, while its short-wavelength tail was observed on the picosecond scale. The absorption band is attributed to the localized holes at $\mathrm{Tl}^{+}$that are stabilized by some defects. In contrast, no absorption band attributable to localized electron centers was observed, indicating that while hole transport is hindered by defects, electron transport is not.

\section{Introduction}

$\mathrm{TlBr}$ is an indirect-gap semiconductor with direct and indirect gap energies of approximately 3.0 and $2.7 \mathrm{eV}$, respectively. ${ }^{(1-5)}$ Owing to the high atomic numbers of its constituent elements (Tl: $81, \mathrm{Br}: 35$ ) and the consequent high interaction probability with high-energy photons such as X-rays and gamma rays, $\mathrm{TlBr}$ has long been a candidate material for semiconductor detectors of ionizing radiations. ${ }^{(6)}$ Its relatively high band-gap energy is also favorable for use at room temperature. Semiconductor detector applications require single crystals of high purity and quality to obtain an excellent energy resolution. However, the development of such applications is limited by issues such as crystal growth reproducibility.

Thus far, TIBr crystal quality has been evaluated using many analysis methods. Among them, optical characterizations have been extensively studied. For instance, the band structure

${ }^{*}$ Corresponding author: e-mail: koshi@qpc.che.tohoku.ac.jp

https://doi.org/10.18494/SAM.2020.2754 
and exciton properties have been investigated using electroabsorption ${ }^{(7)}$ or electroreflectance spectroscopy. ${ }^{(8)}$ Photoconductivity studies have revealed the transport properties of the optically produced electron-hole pairs. ${ }^{(9-12)}$ Similarly, photoluminescence, cathodoluminescence, and scintillation spectroscopies with steady-state and time-resolved measurements at different temperatures have revealed lattice imperfections such as point defects and dislocations. ${ }^{(13-16)}$

In this study, we employed transient absorption spectroscopy to characterize $\mathrm{TlBr}$ crystals. This is a powerful technique to analyze the dynamics of electron-hole pairs. In a previous study, ${ }^{(14)}$ transient absorption spectra were recorded after $20 \mathrm{~ns}$ of excitation using a pulsed electron beam. In this study, we recorded the transient absorption on the pico- and nanosecond scales and compared the transient absorption characteristics of the crystals that are empirically known to be appropriate for semiconductor detectors with those of crystals known to be inappropriate.

\section{Materials and Methods}

TlBr crystals were grown from raw TlBr powder (Sigma-Aldrich, 99.999\%) after many runs of purification by the travelling molten zone (TMZ) method. We used two types of crystal: the first was obtained from a portion of a crystal boule that is empirically known to be appropriate for semiconductor detectors. This crystal was grown after 322 runs of purification by the TMZ method. The second was obtained from a portion of a crystal boule that is empirically known to be inappropriate for semiconductor detectors. This crystal was grown after 228 runs of purification by the TMZ method. The crystals were obtained from the same portion as those used in our previous study. ${ }^{(13)}$ The thicknesses of the appropriate and inappropriate crystals were 0.611 and $0.616 \mathrm{~mm}$, respectively. The details of the crystal growth have been described in a previous paper. $^{(6)}$ After the crystal growth was completed, all measurements were performed at room temperature in air.

The transient absorption after pulsed beam irradiation in the picosecond scale was observed using a measurement system at the Nuclear Professional School, School of Engineering, The University of Tokyo. The crystals were irradiated with a pulsed electron beam whose energy, pulse duration, and charge were $22 \mathrm{MeV}, 7 \mathrm{ps}$ at full width at half maximum, and $2.5 \mathrm{nC}$, respectively. The transient absorption spectra were recorded using white light pulses as the probe light, which were obtained by focusing the fundamental light (780 nm and $100 \mathrm{fs}$ ) of a Ti-sapphire laser (Coherent Inc., Libra-F-HE) onto a $\mathrm{CaF}_{2}$ single crystal. The intense fundamental light in the probe light was cutoff using a band-stop filter prior to its incidence on the sample. The transmitted light spectra of the samples with and without pulsed electron beam irradiation were measured using a photonic multichannel analyzer (Hamamatsu, PMA-20) to obtain the transient absorption spectra. The incident time of the probe light pulses was controlled using an optical delay line up to $40 \mathrm{~cm}$, which corresponds to the time scan width of $2.6 \mathrm{~ns}$. The signal-to-noise ratio $(\mathrm{S} / \mathrm{N})$ was relatively low, below $450 \mathrm{~nm}$, owing to the low intensity of the transmitted light. The details of the measurement system were described in a previous paper. ${ }^{(17)}$ 
The transient absorption after pulsed electron beam irradiation on the nanosecond time scale was measured using a measurement system at The Institute of Scientific and Industrial Research, Osaka University. The samples were simultaneously irradiated with the pulsed electron beam and a pulsed probe light from a flash lamp (Takasago Ltd., YAMA-1500). The energy, duration, and charge of the electron beam were $28 \mathrm{MeV}, \sim 8 \mathrm{~ns}$, and $90 \mathrm{nC}$, respectively. The duration of the pulsed light was several tens of microseconds, which was much longer than that of the pulsed electron beam. Thus, the pulsed light can be considered as a quasi-continuous light. The transmitted light was monochromatized using monochromators for UV-to-visible (UV-VIS; <900 nm) and IR (>900 nm) wavelength regions; the light in each region was detected using a Si photodiode and an InGaAs photodiode, respectively. A digital oscilloscope (Tektronix Inc., DPO7254) was used to record the output signals in the form of transmitted light intensity as a function of time. Transient absorption temporal profiles were obtained as the difference in the transmitted light intensity as a function of time between samples with and without pulsed electron beam irradiation; these profiles at different wavelengths were then used to obtain the transient absorption spectra. The $\mathrm{S} / \mathrm{N}$ was relatively low, below $450 \mathrm{~nm}$, owing to the low intensity of the transmitted light. A small peak at approximately $150 \mathrm{~ns}$ corresponds to the reflection noise. The details of the measurement system have been reported elsewhere. ${ }^{(18,19)}$

\section{Results}

Figure 1 shows the time-resolved transient absorption spectra of the appropriate and inappropriate crystals on the picosecond scale. We observe the short-wavelength edge of the spectra of approximately $430 \mathrm{~nm}$, whose photon energy is between the indirect and direct bandgap energies of approximately 2.7 and $3.0 \mathrm{eV}$, respectively. The photon energy of the short-wavelength edge is higher than the indirect bandgap energy owing to the low absorption coefficient for the optical transition at the indirect gap. The blank wavelength region between 767 and $804 \mathrm{~nm}$ corresponds to the band-stop filter that suppresses the fundamental light of the Ti-sapphire laser. Furthermore, we observe that the spectra are not composed of absorption

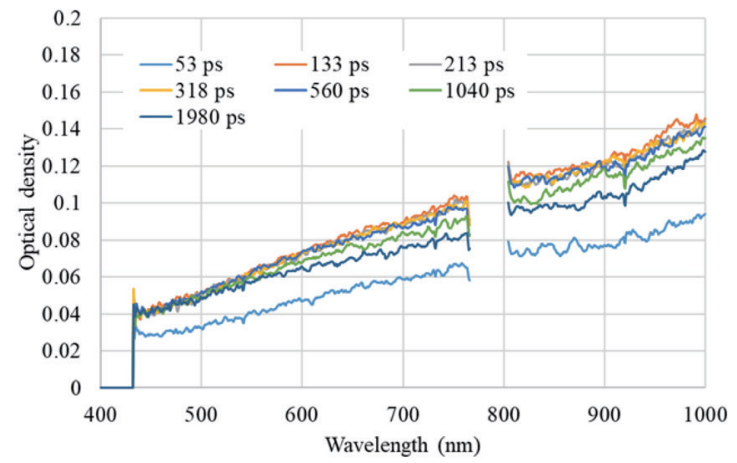

(a)

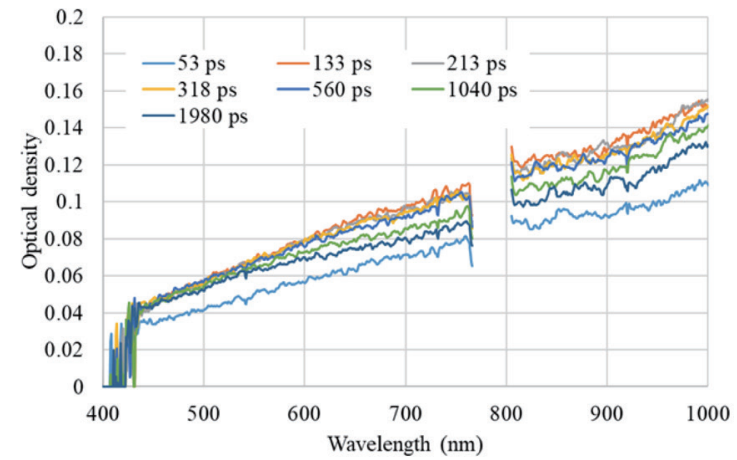

(b)

Fig. 1. (Color online) Time-resolved transient absorption spectra of (a) appropriate and (b) inappropriate crystals on the picosecond scale. 
bands and also do not exhibit an emission band; they are represented by a straight line having a positive slope with wavelength. Figure 2 shows the transient absorption temporal profiles of the appropriate and inappropriate crystals on the picosecond scale. We observe a steep rise within $70 \mathrm{ps}$, although slightly slower than the time resolution of the measurement system at $\sim 25 \mathrm{ps}$. After this rise, the optical density slightly decreases within the time window of $\sim 2 \mathrm{~ns}$; this decrease is more significant for longer wavelengths. The transient absorption spectra in Fig. 1 indicate that the slope becomes gentler over time.

Figure 3 shows the transient absorption spectra of the appropriate and inappropriate crystals on the nanosecond scale. We observe that the spectra at $10 \mathrm{~ns}$ has a complicated shape because of significant noise, while that at $20 \mathrm{~ns}$ and later have a simple structure with a peak at approximately $1160 \mathrm{~nm}$. Figure 4 shows the transient absorption temporal profiles of the appropriate and inappropriate crystals on the nanosecond scale. We observe no significant difference in the decay behavior at different wavelengths, except for the initial slower decay at $1100 \mathrm{~nm}$. Moreover, the decay behavior has not been expressed with a single exponential decay or $1 / t$ dependence, which is generally applied to the decay kinetics of the second order.

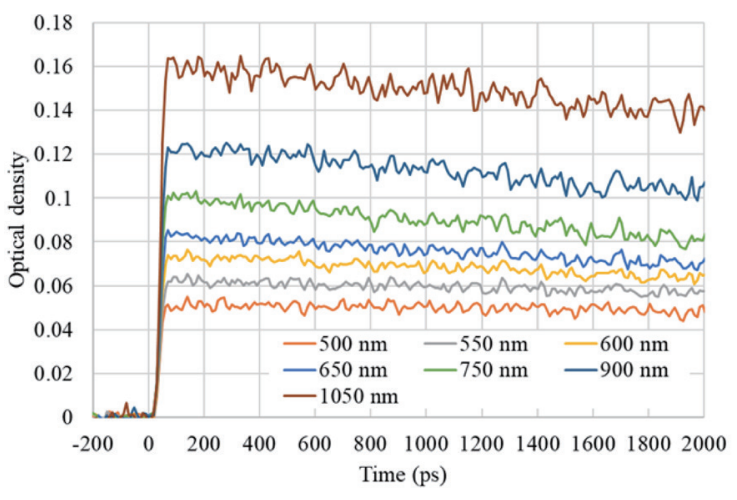

(a)

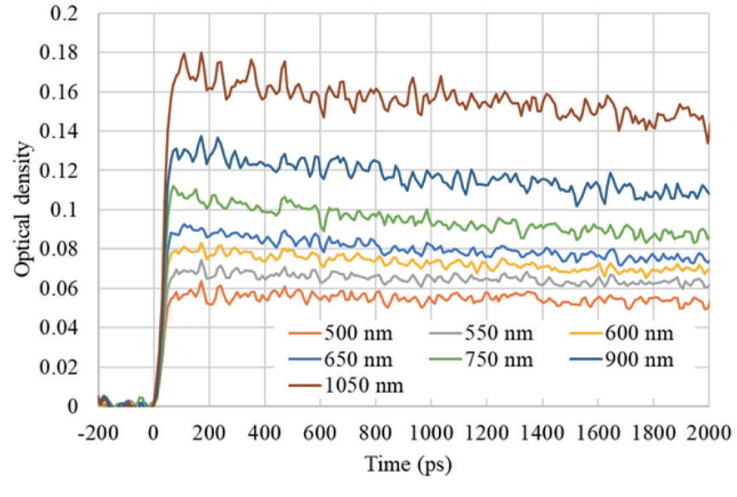

(b)

Fig. 2. (Color online) Transient absorption temporal profiles of (a) appropriate and (b) inappropriate crystals on picosecond scale.

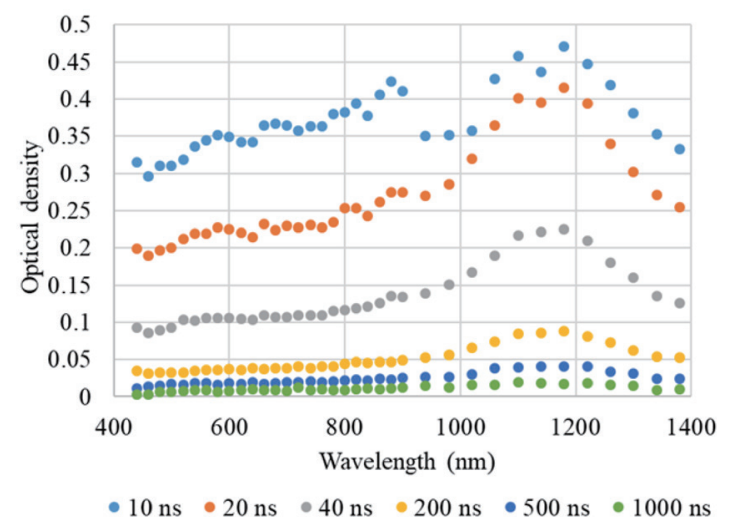

(a)

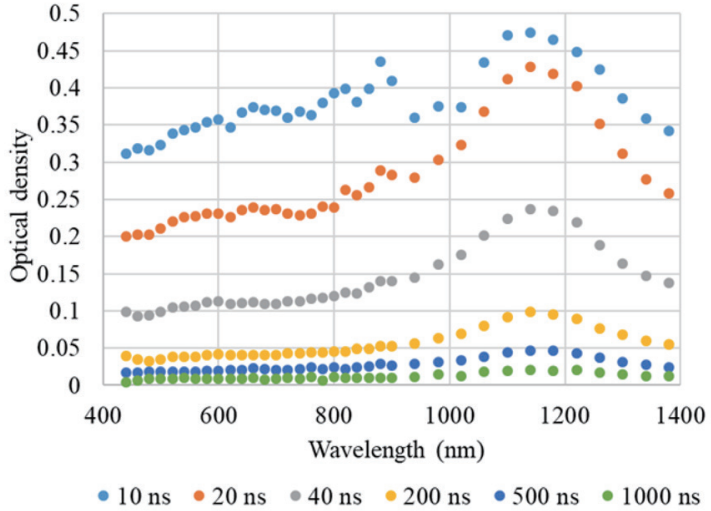

(b)

Fig. 3. (Color online) Time-resolved transient absorption spectra of (a) appropriate and (b) inappropriate crystals on nanosecond scale. 


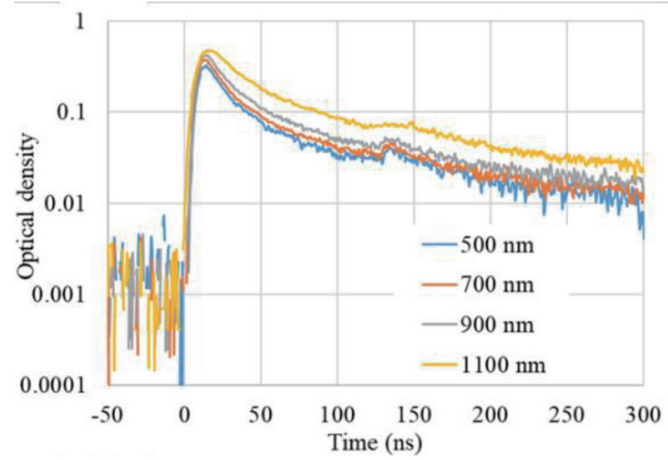

(a)

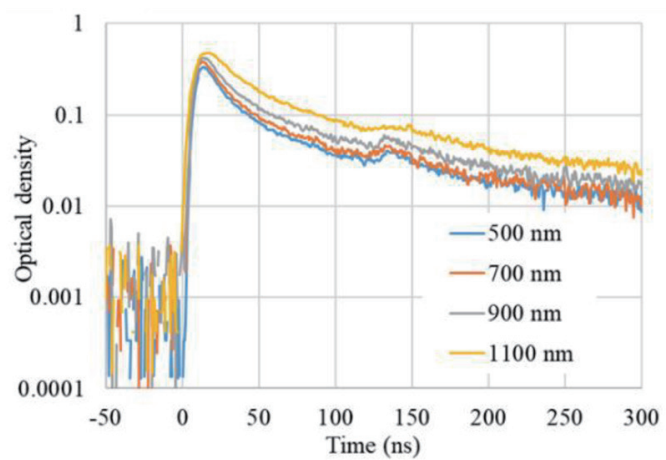

(b)

Fig. 4. (Color online) Transient absorption temporal profiles of (a) appropriate and (b) inappropriate crystals on the nanosecond scale.

\section{Discussion}

The transient absorption spectra at $20 \mathrm{~ns}$ and later have a broad peak at $1160 \mathrm{~nm}$ with a somewhat broad tail toward the short wavelength side down to $\sim 450 \mathrm{~nm}$. Besides, this peak wavelength (i.e., $1160 \mathrm{~nm}$ ) is outside the wavelength range of the picosecond measurements. We observe that the slope of the transient absorption spectra on the picosecond scale exhibits a nearly linear dependence on the wavelength $\lambda$. This dependence contradicts the dependences of free-carrier absorption, which exhibit a wavelength dependence of $\lambda^{1.5}, \lambda^{2}, \lambda^{2.5}$, or $\lambda^{3.5}$, based on the scattering mechanism..$^{(20,21)}$ Moreover, because no absorption band is observed below $1000 \mathrm{~nm}$ both on the pico- and nanosecond time scales, we can safely conclude that the transient absorption spectra on the picosecond scale up to $1000 \mathrm{~nm}$ represent the tail of the absorption band at $1160 \mathrm{~nm}$. A previous paper has already shown the transient absorption band at $1160 \mathrm{~nm}$, which is attributed to holes at the $\mathrm{Tl}$ site $\left(\mathrm{Tl}^{2+}\right)$ stabilized by some defects. ${ }^{(14)}$ The observation of this peak strongly suggests that some hole traps suppress the hole transport in these crystals. However, unlike the crystals reported in the previous paper, ${ }^{(14)}$ no absorption band at $\sim 2.2 \mathrm{eV}$, which is attributed to electron-trapping centers, was observed in the present study. These results indicate that the hole transport of the crystals can be improved by suppressing the generation of related defects, and the electron transport properties of the present crystals are significantly better than those of the crystals analyzed in the previous paper. ${ }^{(14)}$

Considering the dynamics, the steep rise in the transient absorption temporal profiles within $70 \mathrm{ps}$ indicates that some holes are trapped after the production of the electron-hole pairs in less than $70 \mathrm{ps}$. The decay on the picosecond scale was negligible, which indicates that the electron-hole recombination is negligible in this time scale. Furthermore, the decay behavior on the nanosecond scale is not represented by a single exponential decay function nor a $1 / t$ dependence, which indicates that the decay behavior cannot be described by either the firstorder kinetics having a single decay channel or second-order kinetics. Therefore, these results strongly suggest that the decay can be expressed with a sum of multiple exponential decay functions, which corresponds to multiple decay channels. Hence, a plausible origin of the 
multiple decay channels is hole traps of several types. Besides, the decay time scale indicates that the hole traps hinder the hole transport.

The difference between transient absorption behaviors on the pico- and nanosecond scales of the appropriate and inappropriate crystals was negligible, which suggests that the electron and hole trapping behaviors of the crystals are almost similar, although the crystals exhibited significant differences in their photoluminescence and radioluminescence spectra in our previous paper, ${ }^{(13)}$ in which it is strongly suggested that the concentration of dislocation-type defects was significantly different between the two crystals. These results indicate that the two crystals were similar from the viewpoint of the concentration of point defects, whereas they are quite different in the dislocation concentration. As a future prospect, similar measurements under operation as semiconductor detectors would give useful information on the carrier transport.

\section{Conclusions}

We analyzed the transient absorption spectra of $\mathrm{TlBr}$ crystals using pulsed electron beam irradiation and obtained their temporal profiles on the pico- and nanosecond scales. On the basis of the spectral shape on the nanosecond scale, the transient absorption is attributed to the localized holes at $\mathrm{Tl}^{+}$stabilized by some defects. Unlike the previously reported transient absorption, ${ }^{(14)}$ no absorption band of localized electron centers at $2.2 \mathrm{eV}$ was observed in this study. These results indicate that while some defects hinder the hole transport, the electron transport is not hindered by the defects. Moreover, a negligible difference in transient absorption behavior was observed between the appropriate and inappropriate crystals, which indicates that the defect concentrations of electrons and hole-trapping centers are similar.

\section{Acknowledgments}

This research was supported by a Grant-in-Aid for Scientific Research (A) (No. 19H00880, 2019-2022). Part of this research is based on the Cooperative Research Project of the Research Center for Biomedical Engineering, Ministry of Education, Culture, Sports, Science and Technology of Japan. This work was performed under the Cooperative Research Program of "Network Joint Research Center for Materials and Devices", and as a collaborative research project at the Nuclear Professional School, School of Engineering, The University of Tokyo. We thank Professor M. Uesaka and Mr. T. Ueda of The University of Tokyo for providing support in the experiment.

\section{References}

1 J. Nakamura, K. Kobayashi, and A. Fujii: J. Phys. Soc. Jpn. 37 (1974) 1312.

2 R. Farrell, F. Olschner, K. Shah, and M. R. Squillante: Nucl. Instrum. Methods Phys. Res., Sect. A 387 (1997) 194.

3 H. M. Smith III, Y. Zhou, G. Ciampi, H. Kim, L. J. Cirignano, K. S. Shah, E. E. Haller, and D. C. Chrzan: Appl. Phys. Lett. 103 (2013) 091909.

4 R. Z. Bachrach and F. C. Brown: Phys. Rev. B 1 (1970) 818. 
5 R. Shimizu, T. Murahashi, and T. Koda: J. Phys. Soc. Jpn. 33 (1972) 866.

6 K. Hitomi, T. Shoji, and K. Ishii: J. Cryst. Growth 379 (2013) 93.

7 K. Kurita, K. Takiyama, T. Fujita, T. Oda, and A. Fujii: J. Phys. Soc. Jpn. 54 (1985) 4787.

8 R. Shimizu and T. Koda: J. Phys. Soc. Jpn. 38 (1975) 1550.

9 V. Kazukauskas and A. Jurgilaitis: Mol. Cryst. Liq. Cryst. 485 (2008) 77.

10 V. Kazukauskas, A. Ziminskij, G. Davidyuk, V. Bozhko, and G. Mironchuk: Phys. Status Solidi C 6 (2009) 2795.

11 V. Kazukauskas, A. Ziminskij, J. Vaitkus, V. Gostilo, and M. Shorohov: Nucl. Instrum. Methods Phys. Res., Sect. A 607 (2009) 123.

12 V. Kazukauskas, A. Ziminskij, G. Davidyuk, V. Bozhko, and G. Mironchuk: Mol. Cryst. Liq. Cryst. 522 (2010) 82.

13 M. Koshimizu, K. Hitomi, M. Nogami, T. Yanagida, Y. Fujimoto, and K. Asai: Jpn. J. Appl. Phys. 59 (2020) SCCB19.

14 L. Grigorjeva, D. Millers, M. Shorohov, I. S. Lisitskii, M. S. Kuznetsov, S. Zatoloka, and V. Gostilo: Nucl. Instrum. Methods Phys. Res., Sect. A 531 (2004) 197.

15 M. Shorohov, L. Grigorjeva, and D. Millers: Nucl. Instrum. Methods Phys. Res., Sect. A 563 (2006) 78.

16 L. Grigorjeva and D. Millers: Nucl. Instrum. Methods Phys. Res., Sect. B 191 (2002) 131.

17 J. Ma, S. Yamashita, Y. Muroya, Y. Katsumura, and M. Mostafavi: Phys. Chem. Chem. Phys. 17 (2015) 22934.

18 S. Seki, Y. Yoshida, S. Tagawa, and K. Asai: Macromolecules 32 (1999) 1080.

19 K. Kobayashi and S. Tagawa: J. Am. Chem. Soc. 125 (2003) 10213.

20 W. P. Dumke: Phys. Rev. 124 (1961) 1813.

21 H. J. G. Mayer: Phys. Rev. 112 (1958) 298. 